\title{
DANCR Acts as a Diagnostic Biomarker and Promotes Tumor Growth and Metastasis in Hepatocellular Carcinoma
}

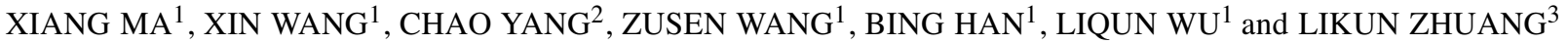 \\ Departments of ${ }^{1}$ Hepatobiliary and Pancreatic Surgery,and ${ }^{2}$ Infectious Diseases, and \\ ${ }^{3}$ Institute of Transplant Medicine, the Affiliated Hospital of Qingdao University, Qingdao, P.R. China
}

\begin{abstract}
Background: Long non-coding RNA differentiation antagonizing non-protein coding RNA (DANCR) is considered to be an oncogene in many cancer types. However, the role of DANCR in diagnosis and progression of hepatocellular carcinoma (HCC) is still unknown. Patients and Methods: In this study, 146 participants including healthy volunteers (HVs) and patients with chronic hepatitis $B(C H B)$, cirrhosis and $H C C$ were recruited. Results: Firstly, quantitative reverse transcription-PCR analysis showed that DANCR was upregulated in tumor tissues and plasma of patients with $H C C$, and its expression was highly correlated with microvascular and liver capsule invasion of HCC. Receiver operating characteristic analysis showed that plasma DANCR exhibited significantly increased discriminatory power for differentiating patients with HCC from HVs and non-HCC patients compared to alpha fetoprotein, which has been used as a biomarker for HCC diagnosis. Furthermore, knockdown of DANCR repressed the $\beta$-catenin pathway and inhibited $H C C$ cell proliferation and metastasis both in vitro and in vivo. Conclusion: This study revealed, for the first time, the importance of DANCR in clinical diagnosis and disease progression of $\mathrm{HCC}$.
\end{abstract}

Hepatocellular carcinoma (HCC) is one of the most common and malignant diseases worldwide (1). Most cases of HCC are associated with viral hepatitis infection and cirrhosis (2). In recent years, abdominal ultrasound, computed tomography, magnetic resonance imaging, liver biopsy and serum biomarkers have become common diagnostic tools for HCC.

Correspondence to: Likun Zhuang, Institute of Transplant Medicine, the Affiliated Hospital of Qingdao University, Qingdao, 266003, P.R. China. Tel: +86 053282917303, e-mail: zlk0823@163.com; or Liqun $\mathrm{Wu}$, Department of Hepatobiliary and Pancreatic Surgery, the Affiliated Hospital of Qingdao University, Qingdao, 266003, P.R. China. Tel: +86 053282917303, e-mail: wuliqun5810@163.com

Key Words: HCC, DANCR, diagnostic biomarker, LncRNA, AFP, cell proliferation and metastasis.
Accordingly, the most common treatments for HCC are liver resection, liver transplantation and radiofrequency ablation. However, the overall survival rate for patients with HCC remains unsatisfactory partly because of delayed diagnosis and recurrence after treatment (3). Therefore, new diagnostic biomarkers and treatment targets are still needed for HCC.

In the past few years, long non-coding RNAs (lncRNAs), with a size of longer than 200 nucleotides, were demonstrated as new diagnostic biomarkers and treatment targets for many diseases, including cancer $(4,5)$. Many lncRNAs have been reported to participate in the pathogenesis of tumors by affecting different biological processes, including cell proliferation, apoptosis, differentiation and migration $(6,7)$. The relatively stable structure of lncRNA in human plasma indicates that lncRNAs might be potential circulating biomarkers for tumor occurrence and progression $(8,9)$. Although more and more lncRNAs have been suggested as important regulators in HCC tumorigenesis $(10,11)$, the roles of the majority of lncRNAs in HCC diagnosis and treatments remain to be explored.

Many studies reported that the lncRNA differentiation antagonizing non-protein coding RNA (DANCR), which was found to suppress the differentiation of epidermal cells (12), plays an important role in tumor progression. For instance, $D A N C R$ promoted invasion of prostate cancer cells by silencing expression of TIMP metallopepitidase inhibitor $2 / 3$ (TIMP2/3) (13). Another study showed that overexpression of $D A N C R$ was associated with advanced tumor progression and poor prognosis in patients with colorectal cancer (14). For HCC, recently, Yuan et al. indicated that DANCR increased stemness features of HCC via de-repression of catenin beta 1 (CTNNB1) (15). However, the diagnostic efficacy of DANCR for HCC and the effects of DANCR on HCC cell proliferation and invasion are still unknown.

In this study, we recruited a cohort of 146 participants including healthy volunteers (HVs) and patients with chronic hepatitis $\mathrm{B}(\mathrm{CHB})$, cirrhosis and $\mathrm{HCC}$, and investigated the potential use of circulating $D A N C R$ in plasma as a diagnostic biomarker for HCC. We also explored the effects of DANCR on $\mathrm{HCC}$ cell proliferation and invasion. Our findings 
Table I. Clinicopathological characteristics of healthy volunteers (HVs) and patients with chronic hepatitis B (CHB), cirrhosis and hepatocellular carcinoma (HCC).

\begin{tabular}{|c|c|c|c|c|c|c|c|}
\hline \multirow[t]{2}{*}{ Clinical factor } & \multirow[t]{2}{*}{ HVs $(n=43)$} & \multirow[t]{2}{*}{$\mathrm{CHB}(\mathrm{n}=29)$} & \multirow[t]{2}{*}{ Cirrhosis $(n=22)$} & \multirow[t]{2}{*}{$\operatorname{HCC}(n=52)$} & \multicolumn{3}{|c|}{$p$-Value (versus $\mathrm{HCC}$ ) } \\
\hline & & & & & HVs & $\mathrm{CHB}$ & Cirrhosis \\
\hline Gender (Male/female) & $26 / 17$ & $15 / 14$ & $13 / 9$ & $34 / 18$ & 0.673 & 0.246 & 0.609 \\
\hline Age (years) & $58.79 \pm 9.67$ & $55.24 \pm 11.89$ & $54.86 \pm 7.62$ & $60.27 \pm 9.27$ & 0.820 & 0.073 & 0.081 \\
\hline $\operatorname{ALT}(\mathrm{U} / \mathrm{l})$ & $17.52 \pm 8.49$ & $78.41 \pm 67.35$ & $29.35 \pm 18.59$ & $67.78 \pm 123.04$ & 0.009 & 0.910 & 0.174 \\
\hline $\operatorname{AST}(\mathrm{U} / \mathrm{l})$ & $18.91 \pm 6.24$ & $61.83 \pm 56.43$ & $34.95 \pm 16.56$ & $69.98 \pm 152.3$ & 0.032 & 0.973 & 0.376 \\
\hline Alkaline phosphatase (U/l) & $87.81 \pm 52.77$ & $73.26 \pm 25.63$ & $90.96 \pm 44.16$ & $120.93 \pm 163.13$ & 0.313 & 0.139 & 0.570 \\
\hline Prothrombin time (s) & $10.45 \pm 1.35$ & $11.71 \pm 1.43$ & $14.74 \pm 4.24$ & $11.11 \pm 1.43$ & 0.338 & 0.506 & 0.000 \\
\hline Albumin $(\mathrm{g} / \mathrm{dl})$ & $37.19 \pm 4.47$ & $37.93 \pm 5.79$ & $33.22 \pm 7.60$ & $37.02 \pm 5.43$ & 0.847 & 1.000 & 0.002 \\
\hline Total bilirubin (mg/dl) & $15.24 \pm 4.60$ & $34.69 \pm 68.36$ & $28.24 \pm 19.08$ & $21.00 \pm 11.25$ & 0.744 & 0.183 & 0.783 \\
\hline
\end{tabular}

ALT: Alanine aminotransferase; AST: aspartate aminotransferase.

provided new insights into the roles of DANCR in HCC tumorigenesis and suggest the potential value of DANCR in the clinical diagnosis of HCC.

\section{Materials and Methods}

Patients and samples. Tissue samples were collected from patients with HCC who underwent partial liver resection at Department of Hepatobiliary and Pancreatic Surgery, the Affiliated Hospital of Qingdao University, from June 2014 to August 2016. Plasma samples were collected from HVs and patients with $\mathrm{CHB}$, cirrhosis and HCC before therapy. Patients with CHB or cirrhosis were diagnosed and selected randomly at Department of Infectious Diseases ranged from June 2015 to August 2016. HCC, CHB and cirrhosis were confirmed by laboratory tests, imaging and histological techniques. The clinicopathological characteristics of patients and HVs are listed in Table I. This work was carried out in accordance with the Declaration of Helsinki for experiments involving humans. This study was also approved by the Ethics Committee of Affiliated Hospital of Qingdao University (application number: 20140105) and all participants provided their informed consent to participate in this study.

Cell culture. HCC cell lines SMMC-7721 and HCCLM3 were obtained from the Cell Resource Center of Shanghai Institutes for Biological Sciences, Chinese Academy of Sciences (Shanghai, China) and cultured in Dulbecco's modified Eagle's medium containing $10 \%$ fetal bovine serum (Gibco, Grand Island, NY, USA) in a humidified atmosphere of $5 \% \mathrm{CO}_{2}$ at $37^{\circ} \mathrm{C}$.

Quantitative reverse transcription-PCR ( $q R T-P C R)$. Total RNAs of tissues or plasma were extracted using Trizol (Invitrogen, Carlsbad, CA, USA). For the extraction of RNA from plasma, 1 pmol SV40 miRNA mimic (Qiagen, Valencia, CA, USA) per $200 \mu$ l plasma was added as spike-in RNA for normalization. After reverse transcription, cDNA was amplified by using SYBR-Green Premix (Takara, Otsu, Japan). The expression of DANCR, vimentin, cyclin D1 (CCND1) and v-myc avian myelocytomatosis viral oncogene homolog $(M Y C)$ in tissues and cell lines were normalized to the expression of $\beta$-actin. The data were analyzed by delta $\mathrm{Ct}$ method. The primers used in this study are listed in Table II.
Transfection and luciferase reporter assay. siRNAs targeting DANCR and negative control (NC) were purchased from GenePharma Company (Shanghai, China). Oligonucleotides were transfected into cells by Hiperfect transfection reagent (Qiagen). TOP/FLASH reporter gene including $\beta$-catenin-binding sites was obtained from Millipore Corporation (Billerica, MA, USA). The reporter constructs were transfected into cells using Lipofectamine 3000 (Invitrogen). Luciferase activity assay was conducted using Dual Luciferase Assay System (Promega, Madison, WI, USA). pRL-TK plasmid (Promega) was used to normalize the transfection efficiency.

Cell proliferation assays. Cell Counting Kit-8 (CCK-8, Dojindo, Kumamoto, Japan) was used to evaluate cell proliferation. Briefly, cells were seeded into a 96-well plate and transfected with corresponding oligonucleotides. At different time points, $10 \mu \mathrm{l}$ CCK-8 solution was added into each well of the plate. The plates were incubated at $37^{\circ} \mathrm{C}$ for $1 \mathrm{~h}$ and the absorbance at $450 \mathrm{~nm}$ was measured. The OD450 value reflected the cell viability.

Transwell invasion assays. Cell invasion assays were conducted using 24-well transwell chambers with $8.0-\mu \mathrm{m}$ pore size polycarbonate membrane (Corning Incorporated, Corning, NY, USA). Cells were seeded on the top side of the membrane precoated with Matrigel (BD, Franklin Lakes, NJ, USA). After incubation in a humidified atmosphere of $5 \% \mathrm{CO}_{2}$ at $37^{\circ} \mathrm{C}$, cells in the upper chamber were removed with cotton swabs. Invaded cells on the lower membrane surface were fixed with methanol and stained with 5\% crystal violet. The cells were counted under a Leica DMI4000B microscope (Leica, Heidelberg, Germany).

HCC xenograft mouse model. DANCR-knockdown lentivirus and the NC lentivirus were purchased from Genechem corporation (Shanghai, China). HCCLM3 cells $\left(5 \times 10^{6}\right)$ infected with the corresponding lentivirus were injected subcutaneously to the posterior flank of the BALB/c nude mice purchased from the Shanghai Institute of Materia Medica (Shanghai, China). One to two weeks later, the subcutaneous tumors were implanted into the livers of new nude mice. About 4 weeks after implantation, the mice were euthanized and the tumors were removed. The tumor volumes were calculated as follows: tumor volume $\left(\mathrm{mm}^{3}\right)=\left(\right.$ length $\times$ width $\left.{ }^{2}\right) / 2$. The lungs of mice were fixed with formaldehyde and the fixed tissues were serial sectioned and stained with hematoxylin-eosin. 
Table II. Primers used in this study.

\begin{tabular}{lll}
\hline Primer & Full name & Sequence \\
\hline$D A N C R$ & $\begin{array}{l}\text { Differentiation antagonizing } \\
\text { non-protein coding RNA } \\
\text { Vimentin }\end{array}$ & $\begin{array}{l}\text { Forward: GCCACTATGTAGCGGGTTTC } \\
\text { Reverse: ACCTGCGCTAAGAACTGAGG }\end{array}$ \\
$V I M$ & Forward: AAAGTGTGGCTGCCAAGAAC \\
$M Y C$ & v-Myc avian myelocytomatosis & Reverse: AGCCTCAGAGAGGTCAGCAA \\
& viral oncogene homolog & Forward: CAAGAGGCGAACACACAACGT \\
Cyclin D1 & Reverse: GGGCCTTTCATTGTTTCCA \\
$A C T B$ & $\beta-A c t i n$ & Forward: CTGGAGGTCTGCGAGGAACA \\
& & Reverse: CTTAGAGGCCACGAACATGCA \\
& & Forward: CATCCTCACCCTGAAGTACCCC \\
\end{tabular}

Then lung metastatic nodules were observed under a Leica DMI4000B microscope (Leica). All animal studies were conducted in the Animal Institute of Qingdao University according to the protocols approved by the Medical Experimental Animal Care Commission of Qingdao University (application number: QDFYMAL-2015122503).

Western blot. Total proteins were extracted from cells transfected with indicated siRNAs using RIPA lysis buffer, separated by sodium dodecyl sulfate-polyacrylamide gel electrophoresis and transferred onto a $0.45 \mu \mathrm{m}$ polyvinylidene fluoride membrane (Millipore). The following primary antibodies were used in the immunoblotting assays: $\beta$-actin and $\beta$-catenin (Cell Signaling Technology, Danvers, MA, USA)

Statistical analysis. Statistical analysis was performed using the SPSS program (version 18.0; Chicago, IL, USA). Data are presented as the mean \pm standard deviation (SD). Statistical significance was calculated by Student's $t$-test, $\chi^{2}$ test or one-way ANOVA followed by Newman-Keul's test. Pearson analysis was used in correlation analysis. Receiver operating characteristic (ROC) and the area under the ROC curve (AUC) was applied to evaluate the diagnostic value and the Youden index was applied to determine the optimal cutoff points of DANCR and alpha fetoprotein (AFP) values in the diagnosis of HCC. The statistical significance of differences between two AUCs was calculated and compared by using Delong's algorithm. A value of $p<0.05$ was considered statistically significant

\section{Results}

DANCR was up-regulated in tumor tissues and plasma of patients with HCC. qRT-PCR analysis showed that the expression levels of $D A N C R$ were significantly higher in tumor tissue than that in paired adjacent non-tumor liver tissue (ANLT) of patients with HCC (Figure 1A). We also found that the plasma DANCR levels in patients with HCC was higher than that in HVs and patients with $\mathrm{CHB}$ and cirrhosis (Figure 1B). These results indicated that DNACR was up-regulated in tumor tissues and plasma in patients with HCC. The correlation of DANCR expression between tumor tissues and plasma from patients with $\mathrm{HCC}$ was then analyzed. The results showed that there was a significant positive relationship between plasma $D A N C R$ and the corresponding tumor tissue DANCR levels (Figure 1C).

Surprisingly, in the 10 patients with HCC who underwent liver resection, plasma $D A N C R$ was significantly lower after surgery than that before surgery (Figure 1D), which suggested that plasma $D A N C R$ might derive from tumor tissues in patients with HCC.

Diagnostic evaluation of plasma DANCR in distinguishing patients with HCC. The diagnostic evaluation of plasma $D A N C R$ in differentiating patients with HCC from controls was conducted by calculating the area under the ROC curve and compared with that of AFP, which has been a useful biomarker for HCC diagnosis. The results showed that AUC for plasma $D A N C R$ was 0.868 which was higher than that for AFP (AUC $=0.744$, Figure 2A and B, Table III) when differentiating patients with $\mathrm{HCC}$ from HVs and non-HCC patients. In view of the fact that AFP was usually up-regulated in patients with chronic hepatitis or cirrhosis and exhibited poor sensitivity for diagnosing HCC (16), we evaluated the power of plasma $D A N C R$ in differentiating patients with $\mathrm{HCC}$ from those with $\mathrm{CHB}$ and cirrhosis. The results showed that the AUC for plasma DANCR was 0.864 which was significantly superior to that for AFP (AUC $=0.650$, Figure $2 \mathrm{C}$ and D, Table IV) when differentiating $\mathrm{HCC}$ patients from patients with $\mathrm{CHB}$ and cirrhosis. At the optimal cutoff values, the sensitivities for plasma DANCR and AFP were $80.8 \%$ and $55.8 \%$, while the specificities were $84.3 \%$ and $76.5 \%$, respectively (Table IV). All these results indicate that plasma $D A N C R$ appears to be a better biomarker than AFP in diagnosing patients with HCC.

Correlation analysis of DANCR levels with clinicopathological characteristics of HCC. In order to explore the correlation between clinicopathological variables and DANCR levels in tumor tissues and plasma, firstly, patients with $\mathrm{HCC}$ were divided into two groups, a high-expression group and a low- 
A

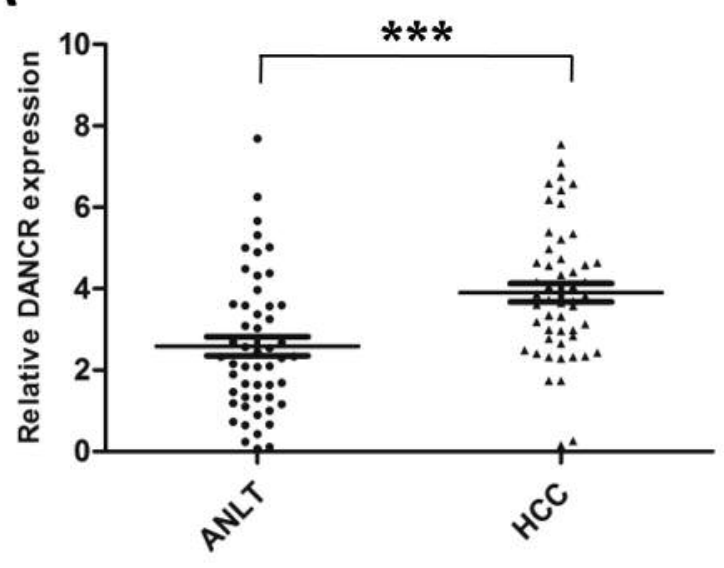

B

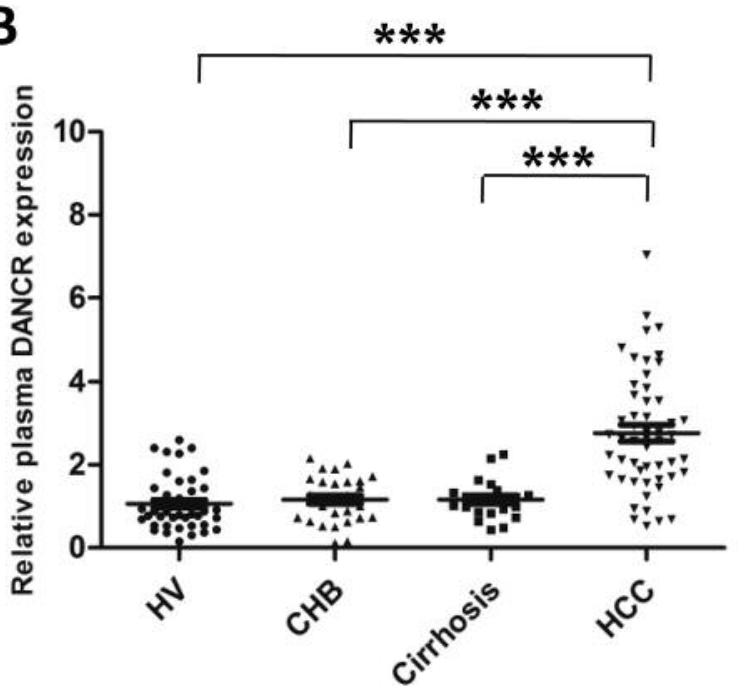

D

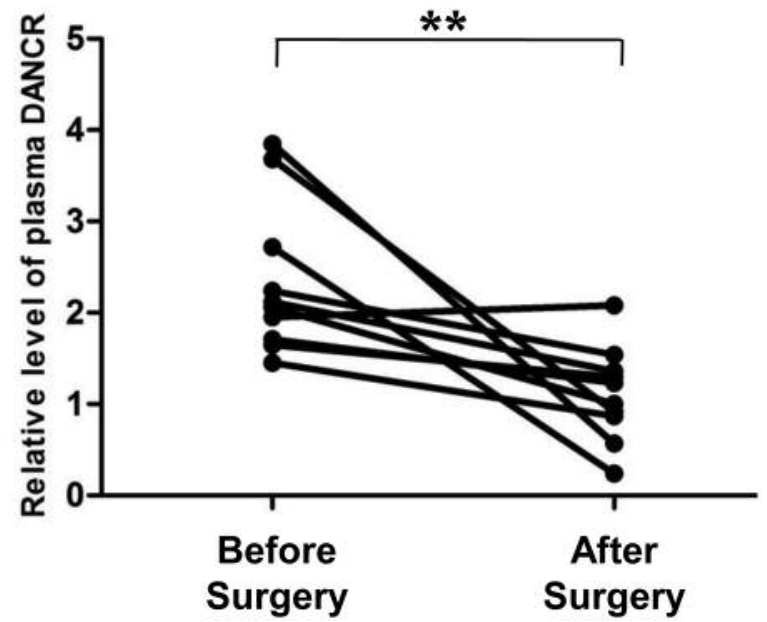

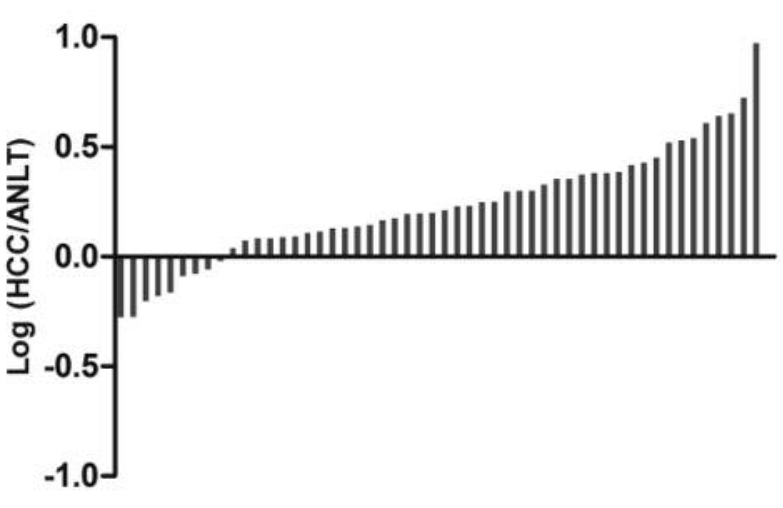

C

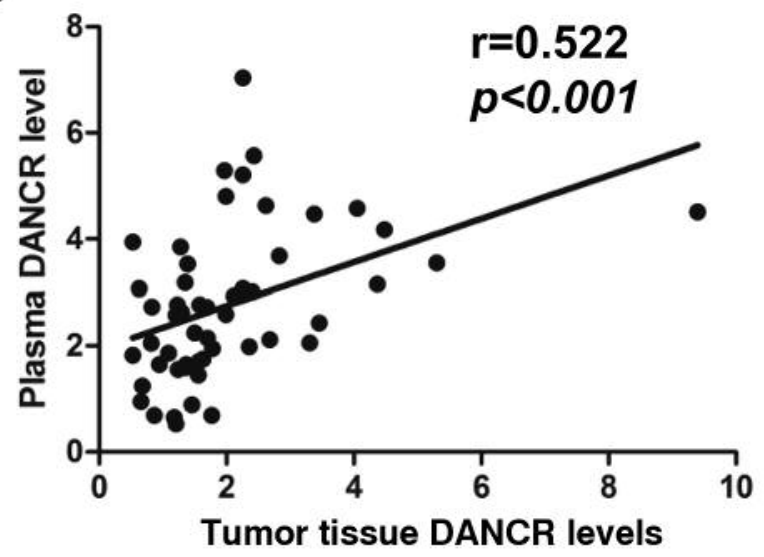

Figure 1. Expression levels of differentiation antagonizing non-protein coding RNA (DANCR) in tissues and plasma from patients with hepatocellular carcinoma (HCC). A: Expression levels of DANCR in 52 pairs of tumor tissues and adjacent non-tumor liver tissues (ANLTS) from patients with HCC were measured by quantitative reverse transcription-PCR and normalized to $\beta$-actin expression. The data were analyzed by delta Ct method. Left panel: DANCR expression levels in tumor tissues and ANLTs were compared with paired Student's t-test. Right panel: The bars represent the relative DANCR expressions with the ratio of its levels in tumor tissue versus ANLT (logarithmic scale). B: Plasma DANCR expressions in healthy volunteers $(H V s)(n=43)$ and patients with chronic hepatitis $B(C H B)(n=29)$, cirrhosis $(n=22)$ and HCC $(n=52)$. DANCR expressions were compared by ANOVA followed by Newman-Keul's test. C: The correlation between tumor tissue and plasma DANCR levels in patients with HCC $(n=52)$. D: Plasma DANCR levels before and after curative resection in patients with HCC $(n=10)$. ** $p<0.01, * * * p<0.001$. 
A

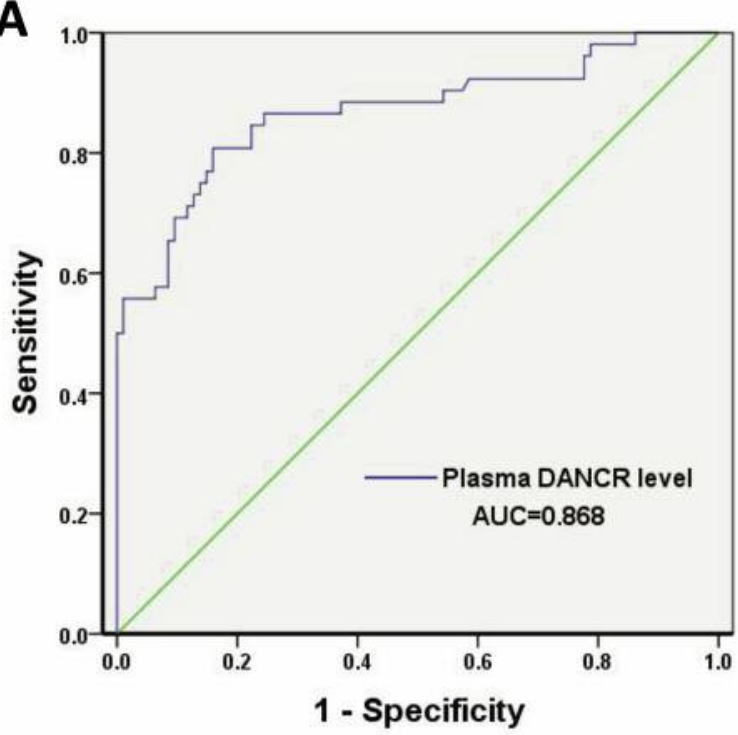

C

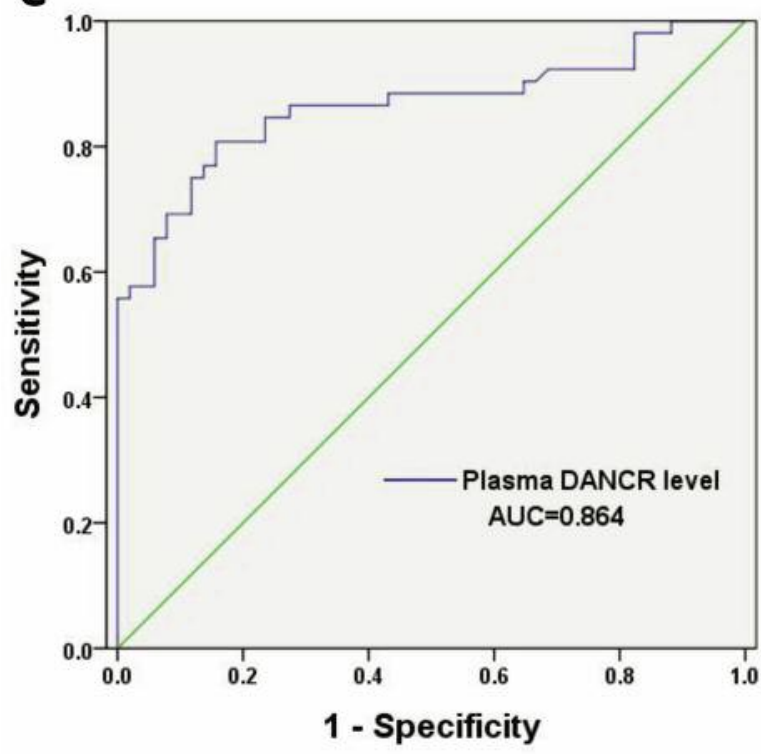

B

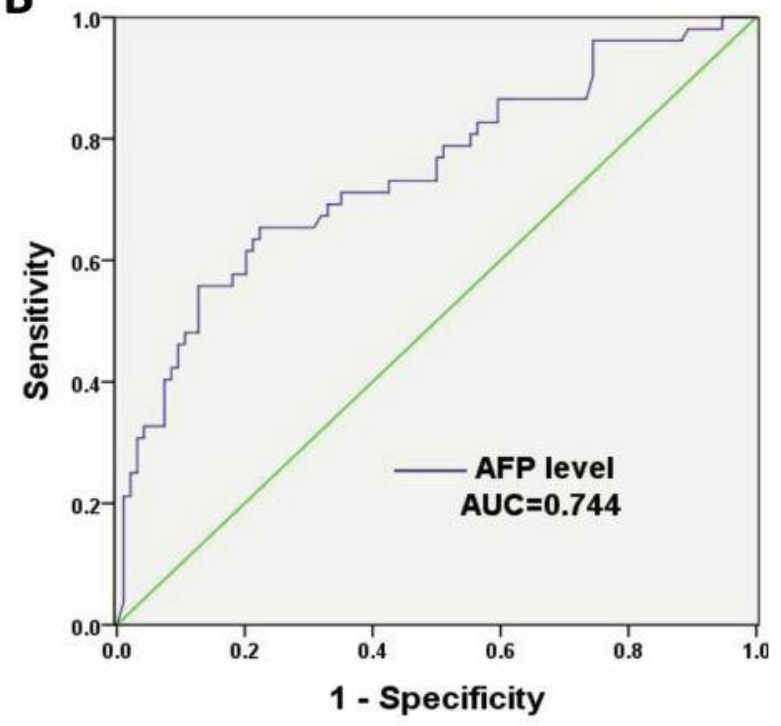

D

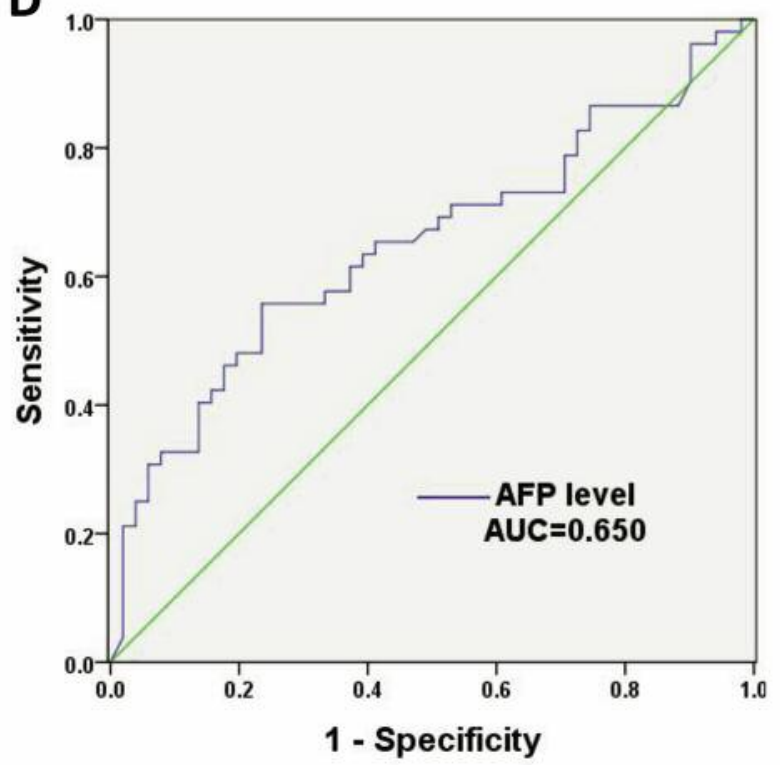

Figure 2. The diagnostic power of plasma differentiation antagonizing non-protein coding RNA (DANCR) and alpha fetoprotein (AFP) for patients with hepatocellular carcinoma (HCC). Receiver operating characteristic (ROC) analysis of plasma DANCR $(A)$ and AFP $(B)$ in differentiating patients with HCC $(n=52)$ from healthy volunteers ( HVs) and patients with chronic hepatitis $B(C H B)$ and cirrhosis $(n=94)$. ROC analysis of plasma $\operatorname{DANCR}(C)$ and $A F P(D)$ in differentiating patients with HCC $(n=52)$ from patients with CHB and cirrhosis $(n=51)$.

Table III. Power of alpha fetoprotein (AFP) and plasma differentiation antagonizing non-protein coding RNA (DANCR) in differentiating patients with hepatocellular carcinoma (HCC) from healthy volunteers (HVs) and patients with chronic hepatitis $B(C H B)$ and cirrhosis.

\begin{tabular}{lcccccc}
\hline HCC versus HVs, CHB and cirrhosis & AUC & Cut-off value & Sensitivity $(\%)$ & Specificity $(\%)$ & Youden index & $p$-Value (versus AFP) \\
\hline AFP & 0.744 & $8.84(\mathrm{ng} / \mathrm{ml})$ & 65.4 & 77.7 & 0.431 & 0.565 \\
Plasma DANCR & 0.868 & $1.65(\Delta \mathrm{Ct})$ & 83.8 & 72.7 & 0.565 \\
\hline
\end{tabular}

AUC: Area under the receiver operating characteristic curve. 
Table IV. Power of alpha fetoprotein (AFP) and plasma differentiation antagonizing non-protein coding RNA (DANCR) in differentiating patients with hepatocellular carcinoma (HCC) from those with chronic hepatitis $B(\mathrm{CHB})$ and cirrhosis.

\begin{tabular}{lcccccc}
\hline HCC versus CHB+cirrhosis & AUC & Cut-off value & Sensitivity (\%) & Specificity (\%) & Youden index & $p$-Value (versus AFP) \\
\hline AFP & 0.650 & $18.97(\mathrm{ng} / \mathrm{ml})$ & 55.8 & 76.5 & 0.323 & 0.651 \\
Plasma DANCR & 0.864 & $1.64(\Delta \mathrm{Ct})$ & 80.8 & 84.3 & 0.3 & 0.001 \\
\hline
\end{tabular}

expression group, according to the DANCR level in tumor tissues (Table V). Correlation analysis indicated that there were more patients with poor tumor differentiation, microvascular invasion and liver capsule invasion in the high expression group.

Patients with HCC were then once more divided into two groups according to the cutoff value of plasma DANCR level for differentiating patients with HCC from HVs and nonHCC patients. Correlation analysis showed that there was a higher proportion of patients with microvascular invasion and liver capsule invasion in the high expression group (Table VI). These results suggest that the DANCR level might be correlated with metastasis of HCC.

Silencing DANCR inhibited HCC cell proliferation and metastasis in vitro and in vivo. In order to explore the biological function of DANCR in HCC cells, HCC cell lines SMMC-7721 and HCCLM3 were transfected with siRNAs for targeting DANCR (siDANCR) and negative control (siNC), respectively. CCK-8 assays were performed to evaluate the effects of DANCR on HCC cell proliferation. Compared with the control group transfected with siNC, transfection of both SMMC-7721 cells and HCCLM3 cells with siDANCR led to a significant decrease in cell viability (Figure $3 \mathrm{~A}$ and $\mathrm{B}$ ). Transwell assays with matrigel were conducted to explore the effects of DANCR on HCC cell invasion. The results indicated that knockdown of $D A N C R$ inhibited the invasive activity of both SMMC-7721 cells and HCCLM3 cells (Figure 3C and D). These results together suggest that silencing DANCR inhibits the proliferation and invasion of HCC cells in vitro.

We also explored the function of DANCR in vivo using a HCC xenograft mouse model. The results showed that knockdown of DANCR in the xenografted tumor cells led to smaller tumor volumes than that in xenografted control cells (Figure 3E). Moreover, the lung metastasis rate decreased from $100 \%$ in the control group to $33.3 \%$ in the group with $D A N C R$ knockdown (Figure $3 \mathrm{~F}$ ). These results suggest an important role for $D A N C R$ knockdown in inhibition of HCC growth and metastasis in vivo.

Silencing DANCR repressed $\beta$-catenin pathway in HCC cells. Previous reports indicated that the activation of $\beta$ catenin pathway promoted tumor cell proliferation and
Table V. Clinicopathological characteristics of patients with hepatocellular carcinoma (HCC) categorized according to the differentiation antagonizing non-protein coding RNA (DANCR) level of tumor tissues.

\begin{tabular}{|c|c|c|c|}
\hline \multirow[t]{2}{*}{ Characteristic } & \multicolumn{2}{|c|}{$\begin{array}{c}D A N C R \text { level of } \\
\text { tumor tissues }\end{array}$} & \multirow[t]{2}{*}{$p$-Value } \\
\hline & $\begin{array}{c}\text { Low }(\mathrm{n}=23) \\
(\mathrm{HCC} / \\
\text { ANLT } \leq 1.5)\end{array}$ & $\begin{array}{l}\text { High }(n=29) \\
(\mathrm{HCC} / \\
\text { ANLT }>1.5)\end{array}$ & \\
\hline Age $(\leq 60 />60$ years $)$ & $9 / 14$ & $13 / 16$ & 0.680 \\
\hline Gender (male/female) & $17 / 6$ & $17 / 12$ & 0.250 \\
\hline Differentiation (well, moderate/poor) & $12 / 11$ & $6 / 23$ & 0.018 \\
\hline Tumor size $(>5 / \leq 5 \mathrm{~cm})$ & $18 / 5$ & $19 / 10$ & 0.341 \\
\hline Number of tumors $(1 / \geq 2)$ & $21 / 2$ & $24 / 5$ & 0.370 \\
\hline TNM stage (I, II/III, IV) & $18 / 5$ & $21 / 8$ & 0.629 \\
\hline Vascular invasion $(-/+)$ & $19 / 4$ & $12 / 17$ & 0.003 \\
\hline Invasion of liver capsule $(-/+)$ & $16 / 7$ & $11 / 18$ & 0.023 \\
\hline Hepatitis B $(-/+)$ & $10 / 13$ & $13 / 16$ & 0.922 \\
\hline Cirrhosis $(-/+)$ & $12 / 11$ & $12 / 17$ & 0.438 \\
\hline
\end{tabular}

ANLT: Adjacent non-tumor liver tissues. Bold numbers indicate significant differences $(p<0.05)$.

Table VI. Clinicopathological characteristics of patients with hepatocellular carcinoma (HCC) categorized according to the plasma differentiation antagonizing non-protein coding RNA (DANCR) level.

\begin{tabular}{|c|c|c|c|}
\hline \multirow[t]{2}{*}{ Characteristic } & \multicolumn{2}{|c|}{ Plasma DANCR level } & \multirow[t]{2}{*}{$p$-Value } \\
\hline & $\begin{array}{l}\text { Low }(n=11) \\
(\Delta \mathrm{Ct} \leq 1.65)\end{array}$ & $\begin{array}{c}\operatorname{High}(\mathrm{n}=41) \\
(\Delta \mathrm{Ct}>1.65)\end{array}$ & \\
\hline Age $(\leq 60 />60$ years $)$ & $4 / 7$ & $18 / 23$ & 0.653 \\
\hline Gender (male/female) & $7 / 4$ & $27 / 14$ & 0.891 \\
\hline Differentiation (well-moderate/poor) & $6 / 5$ & $12 / 29$ & 0.118 \\
\hline Tumor size $(>5 / \leq 5 \mathrm{~cm})$ & $8 / 3$ & $29 / 12$ & 0.897 \\
\hline Number of tumors $(1 / \geq 2)$ & $10 / 1$ & $35 / 6$ & 0.632 \\
\hline TNM stage (I-II/III-IV) & $7 / 4$ & $32 / 9$ & 0.327 \\
\hline Vascular invasion $(-/+)$ & $10 / 1$ & $21 / 20$ & 0.017 \\
\hline Invasion of liver capsule $(-/+)$ & $10 / 1$ & $17 / 24$ & 0.004 \\
\hline Hepatitis B $(-/+)$ & $4 / 7$ & $19 / 22$ & 0.554 \\
\hline Cirrhosis $(-/+)$ & $6 / 5$ & $18 / 23$ & 0.530 \\
\hline
\end{tabular}

ANLT: Adjacent non-tumor liver tissues. Bold numbers indicate significant differences $(p<0.05)$. 
A

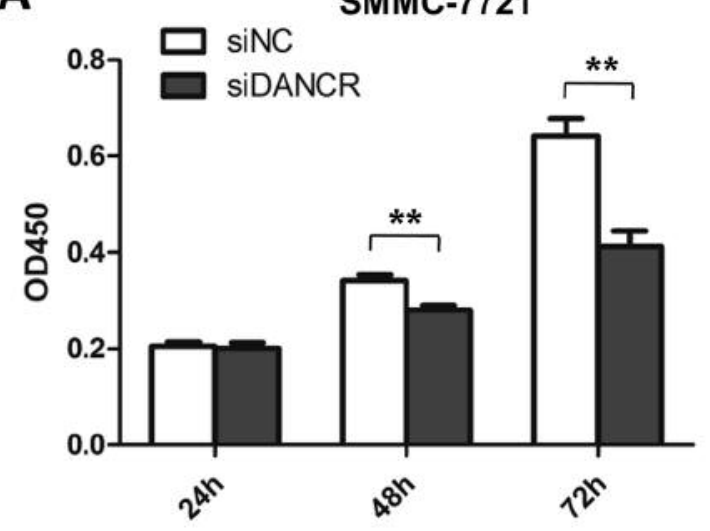

C

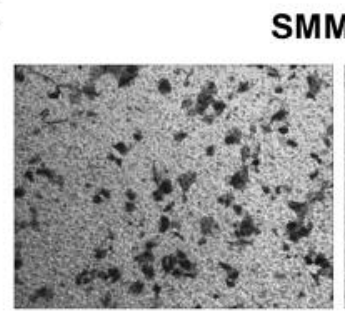

siNC

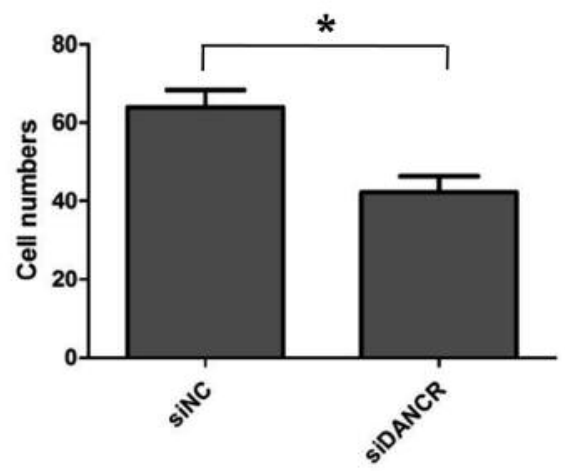

$\mathbf{E}$

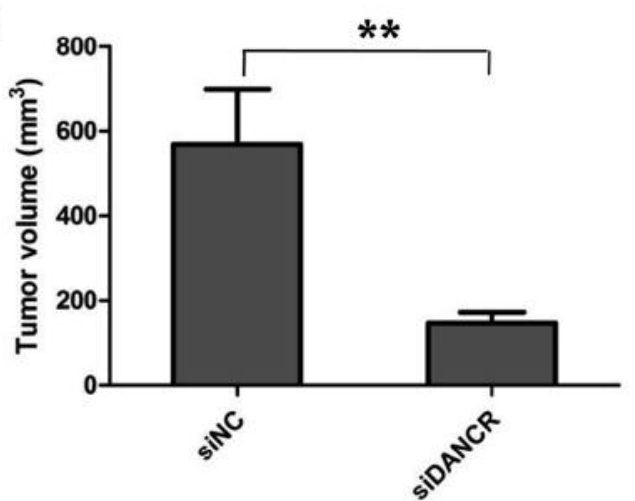

B

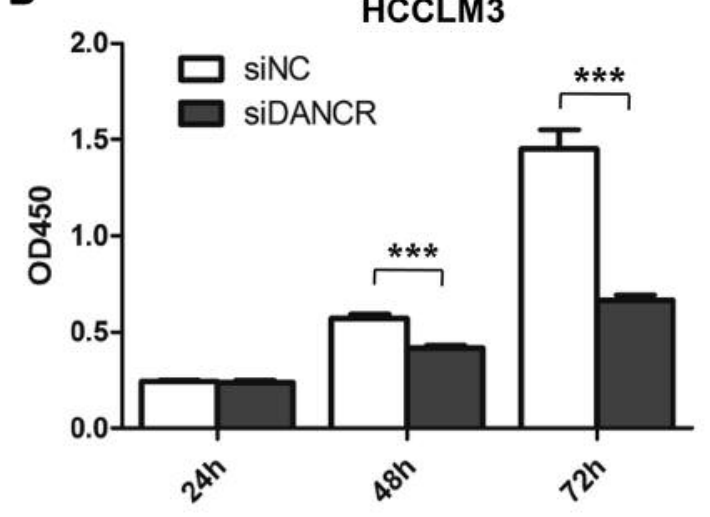

D

HCCLM3

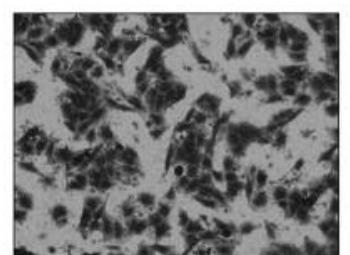

siNC

SiDANCR

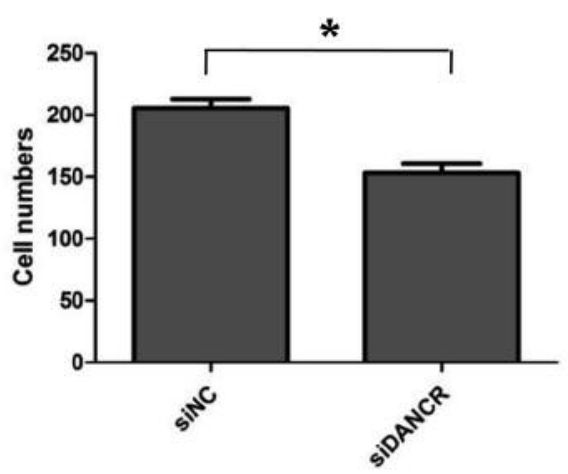

$\mathbf{F}$

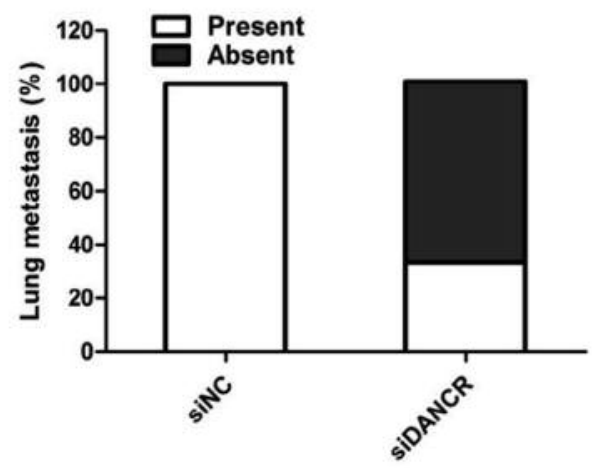

Figure 3. Effects of differentiation antagonizing non-protein coding RNA (DANCR) knockdown on hepatocellular carcinoma (HCC) cell proliferation (A, $B)$ and metastasis $(C, D)$ in vitro and in vivo. CCK-8 analysis of SMMC-7721 (A) and HCCLM3 (B) cells transfected with negative control siRNA (siNC) or siDANCR, respectively. Transwell invasion assays of SMMC-7721 cells (C) and HCCLM3 cells (D) transfected with siNC or siDANCR, respectively. The number of cells passing through the membrane was counted and compared. E: The sizes of local liver tumors of HCC mouse model induced by using HCCLM3 cells infected with siNC or siDANCR lentivirus were calculated and compared with unpaired Student's t-test. Results are presented as the mean \pm S.D. $(n=6) . F$ : The percentage of mice with and without lung metastatic nodules was calculated. ${ }^{*} p<0.05$, ** $p<0.01, * * * p<0.001$. 

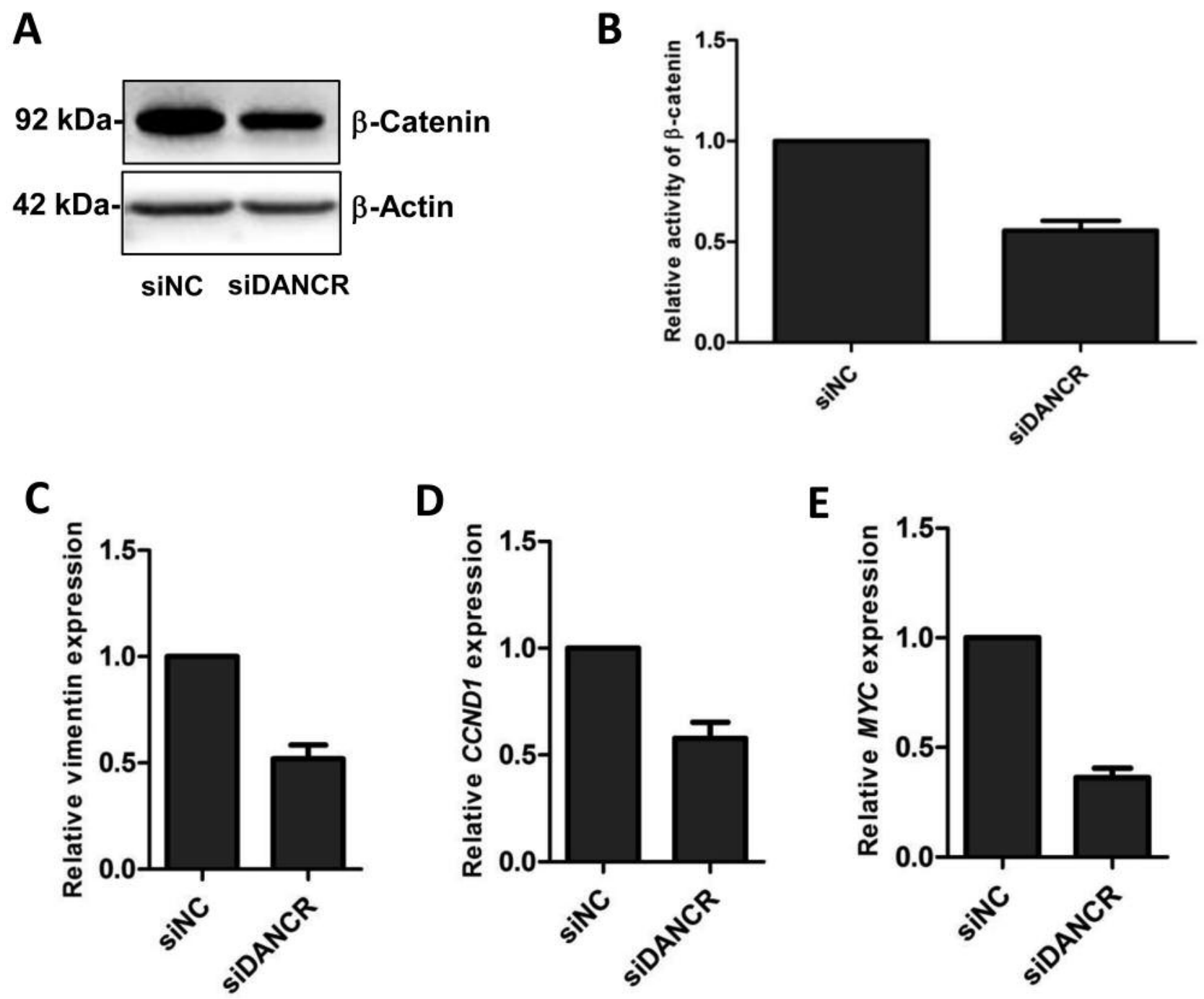

Figure 4. Effects of differentiation antagonizing non-protein coding RNA (DANCR) knockdown on $\beta$-catenin signaling pathway in hepatocellular carcinoma (HCC) cells. A: Representative western blot analysis of $\beta$-catenin in SMMC-7721 cells transfected with negative control siRNA (siNC) or siDANCR. B: Reporter gene analysis of $\beta$-catenin activity in SMMC-7721 cells with siNC or siDANCR transfection. Data were normalized to the activity of $\beta$-catenin in cells with siNC transfection. Results are presented as the mean $\pm S . D .(n=3)$. Quantitative reverse transcription-PCR analysis of vimentin $(C)$, cyclin D1 (CCND1) (D) and v-myc avian myelocytomatosis viral oncogene homolog (MYC) (E) expression in SMMC-7721 cells transfected with siNC or siDANCR. Data were normalized to the corresponding gene expression levels in cells transfected with siNC. Results are presented as the mean \pm S.D. $(n=3)$.

metastasis (17). In investigating the molecular basis for the effects of DANCR in HCC cells, western blot analysis showed that $\beta$-catenin expression levels were lower in SMMC-7721 cells transfected with siDANCR than that in control cells (Figure 4A). When $\beta$-catenin reporter gene and siDANCR were transfected together into SMMC-7721 cells, we found that the activity of $\beta$-catenin was significantly reduced by siDANCR (Figure 4B). Further analysis showed that genes downstream of $\beta$-catenin, namely vimentin, $C C N D 1$ and $M Y C$, were all down-regulated by siDANCR in
SMMC-7721 cells (Figure 4C-E). These results strongly suggest that silencing of $D A N C R$ inhibits $\beta$-catenin signaling in HCC cells.

\section{Discussion}

Although great advances have been achieved in HCC diagnosis in recent years, many patients are still diagnosed with $\mathrm{HCC}$ at an advanced stage and the overall 5-year survival rate of HCC remains very low (3). Currently, AFP is one of 
the most common serum biomarkers for HCC diagnosis (18). However, AFP was reported to exhibit poor diagnostic effectiveness for HCC (16). Considering these reasons, it is necessary to search for a more reliable strategy for HCC diagnosis. In this study, we found that the level of plasma $D A N C R$ was much higher in patients with HCC than that in $\mathrm{HVs}$ and non-HCC patients, and further ROC analysis showed that the diagnostic performance of plasma $D A N C R$ was better than that of AFP in discriminating patients with HCC from HVs and non-HCC patients. These results suggest that plasma $D A N C R$ would be a more useful biomarker than AFP.

Recently, several studies have shown the positive correlation of lncRNAs in plasma and tumor tissues in patients with cancer $(9,19)$. In this study, we also found that plasma $D A N C R$ levels exhibited a positive relationship with tumor tissue $D A N C R$ levels. Combined with the finding that plasma $D A N C R$ levels of patients with HCC were much lower after surgery than before surgery, plasma $D A N C R$ might derive from tumor tissues. However, the in-depth mechanism underlining the positive correlation of DANCR between plasma and tumor tissues remains unknown. The transport of lncRNAs between tumor tissues and plasma might be conducted by means of some kind of microparticles such as exosomes. We would try to explore this hypothesis in future study.

Many reports have indicated that the deregulation of lncRNAs plays important roles in occurrence and progression of many cancer types by regulating tumor cell proliferation and invasion. Our previous study indicated that lncRNA $\mathrm{X}$ inactive specific transcript (XIST) was down-regulated in female patients with HCC and inhibited HCC cell proliferation and metastasis (17). Another lncRNA lung adenocarcinoma associated transcript 1 (LUADT1) was reported to suppress p27 expression and promote lung adenocarcinoma cell proliferation (7). In this study, our results indicated that down-regulation of $D A N C R$ inhibited HCC cell proliferation and invasion in vitro and in vivo. In agreement with previous reports for prostate cancer and colorectal cancer $(13,14), D A N C R$ may also act as an oncogene in HCC progression. Numerous oncogenes or tumor-suppressor genes have been confirmed as targets for lncRNAs in many cancer types, including HCC $(20,21)$. LncRNAs can affect cellular behavior by diverse mechanisms, including direct interaction with DNA, microRNA and protein, and through genetic diversity. For example, the interaction between $m i R-141$ and lncRNA $H 19$ was found to regulate gastric cancer cell proliferation and migration (22). Oncofetal lncRNA plasmacytoma variant translocation 1 (PVT1) promoted proliferation and stem cell-like property of HCC cells by interacting with nucleolar protein 2 (NOP2) (23). In this study, $\beta$-catenin protein levels and the $\beta$-catenin downstream genes vimentin, $C C N D 1$ and $M Y C$ were all down-regulated by siDANCR in HCC cells. However, the specific mechanism for the regulatory function of $D A N C R$ remains to be further explored.

In summary, our results showed for the first time that lncRNA $D A N C R$ was up-regulated in tumor tissues and plasma of patients with $\mathrm{HCC}$, and plasma DANCR might be a useful biomarker for HCC diagnosis. Functionally, knockdown of DANCR inhibited HCC cell proliferation and invasion by suppressing $\beta$-catenin signaling. Our study revealed important roles of DANCR in HCC diagnosis and progression.

\section{Conflicts of Interest}

The Authors declare no conflicts of interest.

\section{Acknowledgements}

This work was supported by grants from the National Natural Science Foundation of China [grant number: 81501812] to LZ, the Natural Science Foundation of Shandong Province [grant number: BS2015SW013] to LZ and the Funding for Excellent Departments (Qingdao University) to LW.

\section{References}

1 Jemal A, Bray F, Center MM, Ferlay J, Ward E and Forman D: Global cancer statistics. CA Cancer J Clin 61: 69-90, 2011.

2 El-Serag HB: Epidemiology of viral hepatitis and hepatocellular carcinoma. Gastroenterology 142: 1264-1273 e1261, 2012.

3 Earl TM and Chapman WC: Conventional surgical treatment of hepatocellular carcinoma. Clin Liver Dis 15: 353-370, vii-x, 2011.

4 Qi P, Xu MD, Ni SJ, Huang D, Wei P, Tan C, Zhou XY and Du $\mathrm{X}$ : Low expression of LOC285194 is associated with poor prognosis in colorectal cancer. J Transl Med 11: 122, 2013.

5 Chen T, Xie W, Xie L, Sun Y, Zhang Y, Shen Z, Sha N, Xu H, $\mathrm{Wu} \mathrm{Z}, \mathrm{Hu} \mathrm{H}$ and $\mathrm{Wu} \mathrm{C}$ : Expression of long noncoding RNA lncRNA-n336928 is correlated with tumor stage and grade and overall survival in bladder cancer. Biochem Biophys Res Commun 468: 666-670, 2015.

$6 \mathrm{Hu}$ D, Su C, Jiang M, Shen Y, Shi A, Zhao F, Chen R, Shen Z, Bao J and Tang W: Fenofibrate inhibited pancreatic cancer cells proliferation via activation of p53 mediated by up-regulation of LncRNA MEG3. Biochem Biophys Res Commun 471: 290-295, 2016.

7 Qiu M, Xu Y, Wang J, Zhang E, Sun M, Zheng Y, Li M, Xia W, Feng D, Yin R and Xu L: A novel lncRNA, LUADT1, promotes lung adenocarcinoma proliferation via the epigenetic suppression of p27. Cell Death Dis 6: e1858, 2015.

8 Tong YS, Wang XW, Zhou XL, Liu ZH, Yang TX, Shi WH, Xie HW, Lv J, Wu QQ and Cao XF: Identification of the long noncoding RNA POU $3 F 3$ in plasma as a novel biomarker for diagnosis of esophageal squamous cell carcinoma. Mol Cancer 14: 3, 2015

9 Gao J, Cao R and Mu H: Long non-coding RNA UCA1 may be a novel diagnostic and predictive biomarker in plasma for early gastric cancer. Int J Clin Exp Pathol 8: 12936-12942, 2015. 
10 Quagliata L, Matter MS, Piscuoglio S, Arabi L, Ruiz C, Procino A, Kovac M, Moretti F, Makowska Z, Boldanova T, Andersen JB, Hammerle M, Tornillo L, Heim MH, Diederichs S, Cillo C and Terracciano LM: Long noncoding RNA HOTTIP/HOXA13 expression is associated with disease progression and predicts outcome in hepatocellular carcinoma patients. Hepatology 59: 911-923, 2014

11 Ge Y, Yan X, Jin Y, Yang X, Yu X, Zhou L, Han S, Yuan Q and Yang M: m iRNA-192 [corrected] and miRNA-204 directly suppress lncRNA HOTTIP and interrupt GLS1-mediated glutaminolysis in hepatocellular carcinoma. PLoS Genet 11: e1005726, 2015.

12 Kretz M, Webster DE, Flockhart RJ, Lee CS, Zehnder A, LopezPajares V, Qu K, Zheng GX, Chow J, Kim GE, Rinn JL, Chang HY, Siprashvili Z and Khavari PA: Suppression of progenitor differentiation requires the long noncoding RNA ANCR. Genes Dev 26: 338-343, 2012.

13 Jia J, Li F, Tang XS, Xu S, Gao Y, Shi Q, Guo W, Wang X, He $\mathrm{D}$ and Guo P: Long noncoding RNA DANCR promotes invasion of prostate cancer through epigenetically silencing expression of TIMP2/3. Oncotarget 7: 37868-37871, 2016.

14 Liu Y, Zhang M, Liang L, Li J and Chen YX: Over-expression of IncRNA DANCR is associated with advanced tumor progression and poor prognosis in patients with colorectal cancer. Int J Clin Exp Pathol 8: 11480-11484, 2015.

15 Yuan SX, Wang J, Yang F, Tao QF, Zhang J, Wang LL, Yang Y, Liu H, Wang ZG, Xu QG, Fan J, Liu L, Sun SH and Zhou WP: Long noncoding RNA DANCR increases stemness features of hepatocellular carcinoma by derepression of CTNNB1. Hepatology 63: 499-511, 2016.

16 Daniele B, Bencivenga A, Megna AS and Tinessa V: Alphafetoprotein and ultrasonography screening for hepatocellular carcinoma. Gastroenterology 127: S108-112, 2004.
17 Zhuang LK, Yang YT, Ma X, Han B, Wang ZS, Zhao QY, Wu LQ and Qu ZQ: MicroRNA-92b promotes hepatocellular carcinoma progression by targeting SMAD7 and is mediated by long non-coding RNA XIST. Cell Death Dis 7: e2203, 2016.

18 Chaiteerakij R, Addissie BD and Roberts LR: Update on biomarkers of hepatocellular carcinoma. Clin Gastroenterol Hepatol 13: 237-245, 2015.

19 Zhou X, Yin C, Dang Y, Ye F and Zhang G: Identification of the long non-coding RNA H19 in plasma as a novel biomarker for diagnosis of gastric cancer. Sci Rep 5: 11516, 2015.

20 Xiang JF, Yin QF, Chen T, Zhang Y, Zhang XO, Wu Z, Zhang S, Wang HB, Ge J, Lu X, Yang L and Chen LL: Human colorectal cancer-specific CCAT1-L lncRNA regulates longrange chromatin interactions at the MYC locus. Cell Res 24: 513$531,2014$.

21 Loewen G, Jayawickramarajah J, Zhuo Y and Shan B: Functions of lncRNA HOTAIR in lung cancer. J Hematol Oncol 7: 90, 2014.

22 Zhou X, Ye F, Yin C, Zhuang Y, Yue G and Zhang G: The interaction between MiR-141 and lncRNA-H19 in regulating cell proliferation and migration in gastric cancer. Cell Physiol Biochem 36: 1440-1452, 2015.

23 Wang F, Yuan JH, Wang SB, Yang F, Yuan SX, Ye C, Yang N, Zhou WP, Li WL, Li W and Sun SH: Oncofetal long noncoding RNA $P V T 1$ promotes proliferation and stem cell-like property of hepatocellular carcinoma cells by stabilizing NOP2. Hepatology 60: 1278-1290, 2014. 